\title{
Aplicação do modelo linear na avaliação de dados de estabilidade de medicamento
}

\section{Linear model applied to the evaluation of pharmaceutical stability data}

\author{
Renato Cesar de Souza ${ }^{1}$, Jaqueline Raminelli ${ }^{2}$
}

\section{Resumo}

O prazo de validade na embalagem de um produto fornece ao consumidor a confiança de que o produto ira manter a sua identidade, teor, qualidade e pureza durante todo o período de validade do produto farmacêutico. A definição do prazo de validade na indústria farmacêutica é baseada nos dados do estudo de estabilidade obtidos durante o registro do produto. Dado o exposto, esse trabalho tem o objetivo de aplicar a regressão linear de acordo com os critérios estabelecidos pelo guia de estabilidade ICH Q1E de 2003 para avaliar alguns aspectos de um produto em fase de registro no Brasil. Com esse propósito, a avaliação foi realizada em um centro de pesquisa e desenvolvimento de uma empresa farmacêutica multinacional no Brasil com amostras de três lotes diferentes de um produto composto por dois princípios ativos em dois tipos de embalagem. Baseado nos resultados preliminares obtidos, foi possível observar a diferença na tendência de degradação do produto nas diferentes embalagens e a relação entre as varáveis estudadas, acrescentando o conhecimento ao grupo de trabalho de forma que novos modelos podem ser desenvolvidos e aplicados para outros produtos.

Palavras-chave: Modelo de equação linear, estudo de estabilidade, registro de medicamentos, prazo de validade.

\begin{abstract}
The expiry date on the packaging of a product gives the consumer the confidence that the product will retain its identity, content, quality and purity throughout the period of validity of the drug. The definition of this term in the pharmaceutical industry is based on stability data obtained during the product registration. By the above, this work aims to apply the linear regression according to the guideline ICH Q1E, 2003, to evaluate some aspects of a product undergoing in a registration phase in Brazil. With this propose, the evaluation was realized with the development center of a multinational company in Brazil, with samples of three different batches composed by two active principal ingredients in two different packages. Based on the preliminary results obtained, it was possible to observe the difference of degradation tendency of the product in two different packages and the relationship between the variables studied, added knowledge so new models of linear equations can be applied and developed for other products.
\end{abstract}

Key words: Linear equation model, stability study, medicines registration, expiration date.

\footnotetext{
${ }^{1}$ Aluno do curso de Especialização em Estatística Aplicada à Pesquisa, DSTA/UEL, renatosou@gmail.com

${ }^{2}$ Departamento de Estatística, Universidade Estadual de Londrina - UEL, raminelli@uel.br
} 


\section{Introdução}

O prazo de validade na embalagem de um medicamento proporciona ao consumidor a confiança de que o medicamento irá reter sua identidade, teor, qualidade e pureza ao longo do período de validade do medicamento. A vida útil do produto é normalmente determinada através de dados de estudo de estabilidade (CHOW, 2007), o qual define-se como conjunto de testes projetados para obter informações sobre a estabilidade dos produtos farmacêuticos visando definir sua vida média e período de utilização em embalagem e condições de estocagem especificada (Brasil, 2004), tendo como objetivo, fornecer evidências de como a qualidade de um produto varia com o tempo sob a influência de uma série de fatores como temperatura, umidade e luz (ICH, 2003).

$\mathrm{O}$ produto em estudo é amostrado aleatoriamente e armazenado em condições de temperatura e umidade controlada. Amostras individuais são coletadas após um período de tempo pré determinado e analisados visando obter informações das características físicas, químicas, biológicas e microbiológicas, incluindo aquelas relacionadas a determinados atributos da forma farmacêutica (por exemplo, a taxa de dissolução para formas farmacêuticas sólidas orais). A partir dos resultados obtidos, obtem-se a especificação do produto em avaliação (WESSELS et al., 1997).

Para a determinação do prazo de validade do produto os dados devem ser avaliados de forma a determinar os atributos críticos susceptíveis de influenciar a qualidade e o desempenho do fármaco ou produto. Cada atributo deve ser avaliado separadamente, e uma avaliação global deve ser feita dos resultados com a finalidade de propor um período de reteste ou vida de prateleira. O período de reteste ou vida útil proposto não deve exceder o previsto para qualquer atributo único (ICH, 2003).

No guia de orientação da Conferência Internacional de Harmonização (ICH) de 2003, é indicado que para o registro de um produto, é necessário pelo menos 12 meses de informações sobre o estudo de estabilidade a longo prazo de pelo menos três lotes do produto. A meta de desenvolvimento de um novo medicamento é uma vida útil que seja significativamente superior a 12 meses. Atualmente, 36 meses de vida útil é aceito pelos órgãos reguladores como um tempo de vida máximo para produtos instáveis e 60 meses é aceito como máximo para os produtos que não mudam com o tempo. Isto pode conduzir a uma situação em que a definição de especificações tem que ser feita com base em apenas 12 meses de dados disponíveis ao final do período de pedido de registro. Isto é difícil e precisa de uma estratégia especial (WESSELS et al., 1997).

Um procedimento para avaliação estatística dos dados de estabilidade com a finalidade de determinação da vida útil - não para a estipulação das especificações para as características de qualidade em si é descrita nos guias de orientação $\mathrm{ICH}$. O procedimento também tem sido descrito em detalhes em várias publicações (NORWOOD, 1986; CHOW; SHAO, 1989; BAR, 2003; CHOW, 2007; LEE; WU; LEE, 2010).

O presente trabalho utiliza a análise estatística para avaliar um PRODUTO X, com dois principios ativos designados como COMPOSTO A de concentração $15 \mu \mathrm{g} /$ comp e COMPOSTO B de concentração $60 \mu \mathrm{g} / \mathrm{comp}$ para avaliar e comparar a eficiência de duas embalagens diferentes na proteção desse produto aos agentes de degradação. Esse produto foi desenvolvido no centro de pesquisa e desenvolvimento brasileiro de uma empresa farmacêutica multinacional de genéricos e encontra-se em fase de registro. Esse grupo de pesquisa nunca aplicou esse tipo de análise, sendo assim, pretende-se adicionalmente com esse trabalho, fazer a inferência do prazo de validade do produto em estudo e disponibilizar a essa empresa uma forma prática de avaliação dos dados de estabilidade de produtos em fase de desenvolvimento. 


\section{Materiais e Métodos}

Estudo de caso

O estudo foi realizado em uma empresa farmacêutica multinacional de genéricos, considerando uma amostra de três lotes do produto $\mathrm{X}$ composto por dois princípios ativos, designados como composto A de concentração $15 \mu \mathrm{g} /$ comp e composto B de concentração $60 \mu \mathrm{g} / \mathrm{comp}$, que encontra-se em 18 meses de estudo de estabilidade de longa duração Brasil $\left(30^{\circ} \mathrm{C} / 75 \%\right.$ U.R.) de acordo com resolução RE n. ${ }^{\circ} 398$ de 2004 da ANVISA.

Os comprimidos foram armazenados em dois tipos de embalagem (PVDC 90 e PVDC 90 + Flow Pack (FP)) e o teor dos dois princípios ativos e as substâncias relacionadas totais dos lotes foram avaliados nos tempos $0,3,6,9,12$ e 18 meses. A especificação de estabilidade é de $90 \%$ a $105 \%$.

\section{Modelo Matemático}

O prazo de validade foi estimado de acordo com o modelo descrito no guia de estabilidade ICH Q1E de 2003.

Seja y(t) o teor médio observado, expresso em porcentagem do valor rotulado, de um lote em um mês t e considerando que a relação entre o teor e o tempo é linear, a regressão linear de cada lote pode ser representada pela seguinte equação,

$$
Y_{i}(t)=\alpha+\beta t_{i}+\epsilon_{i}, \quad i=1, \ldots, n
$$

em que $\alpha$ é o intercepto e $\beta$ é o coeficiente angular, o qual é a taxa de degradação por unidade de tempo; e $\epsilon_{i}$ é a variável aleatória com distribuição normal de média zero e variância $\sigma^{2}$.

O prazo de validade para um único lote pode ser estimado através do ponto onde a linha inferior do intervalo de confiança (95\%) da regressão atinge o limite inferior da especificação do produto. Assumindo que $(\hat{\alpha}, \hat{\beta})$ são os estimadores de $(\alpha$, $\beta)$ baseados nos dados de estabilidade de $\left(y_{i}, t_{i}\right)$ da equação 1 para qualquer tempo fixo. Uma linha inferior do intervalo de confiança (95\%) é dado por:

$$
L(x)=\hat{\alpha}+\hat{\beta} x-\hat{\sigma} t_{n-2} \sqrt{\frac{1}{n}+\frac{x-y}{s_{x x}}}
$$

em que $t_{n-2}$ é o quantil 0,95 da distribuição t com n-2 graus de liberdade, $\bar{x}$ a média dos $x_{i}{ }^{\prime} s$ e $S_{x x}$ o desvio padrão da regressão. A equação 2 foi utilizada para cada lote visando avaliar qual o princípio ativo determinante na degradação e qual a embalagem mais eficiente na proteção ao produto. Através da análise de variância (ANOVA) das regressões, foi possível verificar também a existência de dependência do teor em relação ao tempo através do teste de significância da taxa de degradação por unidade de tempo.

Do ponto de vista estatístico, a associação dos dados obtidos de diferentes lotes armazenados em condições semelhantes (condições de armazenamento, embalagem) em uma estimativa única é apropriada apenas se os dados forem comprovadamente homogêneos.

Este teste é feito por meio do cálculo da análise de covariância (ANCOVA) (WESSELS et al., 1997). A ANCOVA pode ser utilizada para determinar se as linhas de regressão obtidas dos diferentes lotes têm uma inclinação e um intercepto comuns no tempo zero, onde o tempo é considerado a covariável. AANCOVA também é capaz de estimar a variabilidade dentro de lotes com um grau de liberdade maior do que a análise individual dos lotes quando se tem mais de um lote ou diferentes embalagens. Para o modelo linear de regressões de vários lotes do mesmo medicamento, $\mathrm{o}$ modelo de ANCOVAé,

$$
Y_{i}(t)=\alpha_{0}+\alpha_{i}+\left(\beta_{0}+\beta_{i}\right) t+\epsilon_{\mathrm{it}}, \quad i=1, \ldots, n
$$

onde $Y_{i}(t)$ denota o valor médio observado do $i$-ésimo lote no mês t, $\alpha_{0}$ e $\beta_{0}$ são os interceptos e coeficientes angulares comum a todos os lotes; $\alpha_{1}$ e $\beta_{1}$ são os desvios do intercepto e coeficiente angular individuais do $i$-ésimo lote de $\alpha_{0}$ e $\beta_{0}$ respectivamente; $\varepsilon_{\text {it }}$ são os erros aleatórios do modelo com distribuição $\mathrm{N}\left(0, \sigma^{2}\right)$ (TSONG et al., 2003). 
Em seguida, de acordo com os resultados obtidos, pode-se seguir três caminhos diferentes.

De acordo com guia da ICH Q1E de 2003, para o teste de igualdade de coeficientes angulares e interceptos deve-se trabalhar com um nível de significância de 0.25 .

Todas as análises estatísticas foram feitas utilizando o pacote $S T A B$ do software R versão 2.11.1 (TEAM RDC, 2011).

\section{Desenvolvimento}

Avaliação do teor dos ativos no produto

A Tabela 1 apresenta os resultados de teor (apresentados como porcentagem do valor rotulado) obtidos para os lotes do produto $\mathrm{X}$ durante os 18 meses de estudo de estabilidade

Verifica-se que até o período de 18 meses de estudo de estabilidade, os dois compostos do produto não atingiram o limite inferior da especificação de $90 \%$.

Tabela 1 - Teor dos dois compostos do produto X embalado em PVDC e PVDC +FP em estudo de estabilidade de longa duração Brasil $\left(30^{\circ} \mathrm{C} / 75 \%\right.$ U.R.)

\begin{tabular}{ccrrrrrrrrrrrr}
\hline & \multicolumn{1}{c}{ Composto A } & \multicolumn{1}{c}{ Tempo (mês) } \\
\hline Embalagem & Lote & \multicolumn{1}{c}{0} & 3 & 6 & 9 & 12 & 18 & 0 & 3 & 6 & 9 & 12 & 18 \\
& 1 & 97,9 & 100,6 & 100,7 & 97,0 & 97,4 & 95,3 & 100,3 & 98,9 & 100,0 & 97,6 & 96,6 & 97,6 \\
PVDC & 2 & 101,3 & 102,4 & 102,6 & 98,7 & 99,4 & 97,2 & 103,4 & 101,8 & 101,9 & 99,6 & 99,2 & 98,6 \\
& 3 & 99,5 & 101,5 & 100,7 & 98,0 & 98,6 & 95,9 & 102,3 & 101,0 & 100,3 & 99,2 & 98,1 & 97,5 \\
& $1 A$ & 97,9 & 100,5 & 101,2 & 98,3 & 97,9 & 99,2 & 100,3 & 99,7 & 100,3 & 98,1 & 97,2 & 100,2 \\
PVDC + FP & 2A & 101,3 & 101,5 & 103,3 & 100,0 & 100,4 & 100,6 & 103,4 & 101,7 & 102,7 & 99,9 & 100,1 & 101,8 \\
& 3A & 99,5 & 99,6 & 102,1 & 98,3 & 98,6 & 99,1 & 102,3 & 99,6 & 101,8 & 98,7 & 98,1 & 100,1 \\
\hline
\end{tabular}

As Figuras 1 e 2 correspondem a representação gráfica dos dados apresentados na Tabela 1.

Em síntese os resultados encontrados na Tabela 2 evidenciaram que o prazo de validade estimado do produto embalado em PVDC + FP é superior

Figura 1 - Representação gráfica dos dados de estabilidade do produto embalado em PVDC 90+F

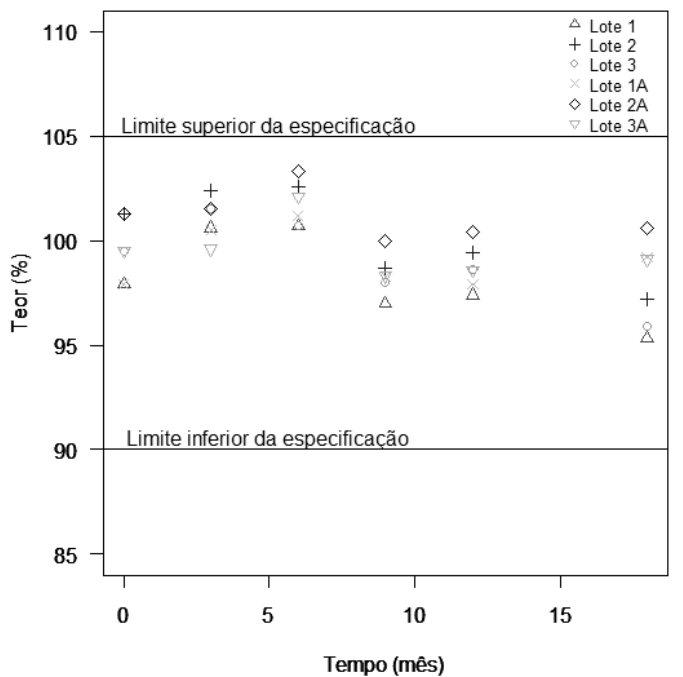

ao produto embalado em PVDC o que condiz com o esperado, visto que a embalagem PVDC + FP fornece uma proteção maior quanto à umidade e oxidação, os quais são os principais agentes responsáveis pela degradação desses ativos.

Figura 2 - Representação gráfica dos dados de estabilidade do produto embalado em PVDC90

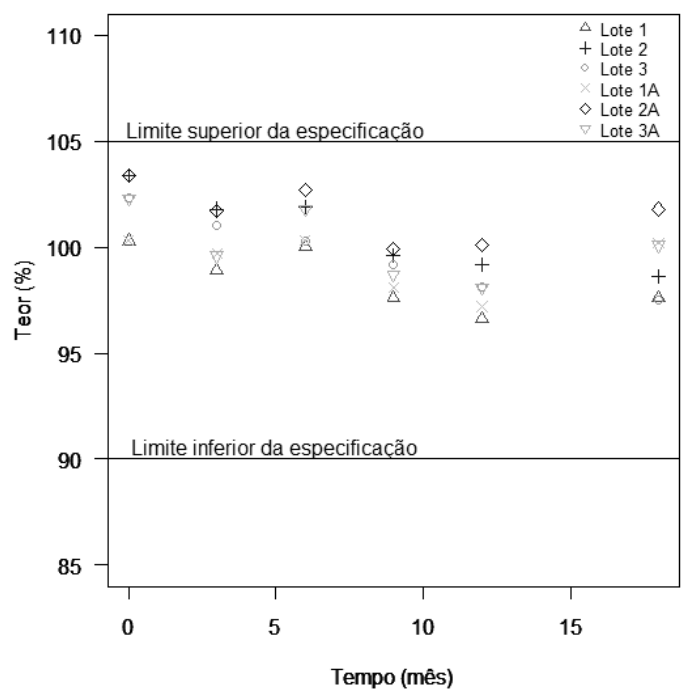


Verifica-se também que o composto determinante para o prazo de validade desse produto é o composto A, por apresentar geralmente uma estimativa de prazo de validade inferior comparado ao composto $\mathrm{B}$, isso devido principalmente a alta variabilidade do composto A que contribui consideravelmente na incerteza da estimativa do prazo de validade. Essa elevada variabilidade pode ser explicada devida a baixa concentração desse composto e a técnica de detecção utilizada, que é muito mais susceptível a variações quando comparada a técnica do composto B.

Ao avaliar o teste de significância dos coeficientes angulares da regressão é possível observar que, com exceção do lote 1 , o coeficiente angular é significativamente diferente de zero $(\mathrm{p}$-valor $<0,05)$, indicando portanto que há uma queda significativa do teor para o produto nessa embalagem. Para o produto embalado em PVDC + FP, pode-se afirmar que o coeficiente angular não é significativamente diferente de zero, indicando assim que não há uma queda do teor quando embalado nessa embalagem, com nível de significância de 5\%. Ao avaliar os valores do coeficiente de determinação $\left(\mathrm{R}^{2}\right)$, observa-se que a queda de teor para o produto embalado em PVDC 90 apresenta uma boa relação linear entre a variável tempo e teor. Entretanto, tem-se uma fraca correlação para o produto embalado em PVDC 90+FP, indicando que a variação nos resultados de teor ao longo do tempo para essa embalagem é fracamente explicada pela variável tempo.

Tabela 2 - Resumo dos modelos lineares individuais dos três lotes embalados (PVDC e PVDC + FP)

\begin{tabular}{|c|c|c|c|c|c|c|c|c|}
\hline Embalagem & Lote & Composto & $\begin{array}{l}\text { Coeficiente } \\
\text { angular }\end{array}$ & $\operatorname{Pr}>F$ & Intercepto & $\mathrm{R}^{2}$ & s & $\begin{array}{c}\text { Prazo } \\
\text { estimado (meses) }\end{array}$ \\
\hline \multirow{6}{*}{ PVDC } & \multirow{2}{*}{1} & $\mathrm{~A}$ & $-0,229$ & 0,1240 & 100,0 & 0,486 & 1,700 & 22 \\
\hline & & B & $-0,176$ & 0,0720 & 99,9 & 0,596 & 1,050 & 30 \\
\hline & \multirow{2}{*}{2} & $\mathrm{~A}$ & $-0,280$ & 0,0357 & 102,5 & 0,708 & 1,320 & 27 \\
\hline & & B & $-0,274$ & 0,0049 & 102,9 & 0,888 & 0,310 & 34 \\
\hline & \multirow{2}{*}{3} & A & $-0,255$ & 0,0045 & 101,1 & 0,676 & 1,280 & 26 \\
\hline & & B & $-0,273$ & 0,0008 & 101,9 & 0,954 & 0,440 & 35 \\
\hline \multirow{6}{*}{$\begin{array}{l}\text { PVDC + Flow } \\
\text { Pack }\end{array}$} & \multirow{2}{*}{$1 \mathrm{~A}$} & $\mathrm{~A}$ & $-0,030$ & 0,7970 & 99,4 & 0,018 & 1,560 & 29 \\
\hline & & B & $-0,060$ & 0,5730 & 99,8 & 0,086 & 1,420 & 34 \\
\hline & \multirow{2}{*}{$2 \mathrm{~A}$} & $\mathrm{~A}$ & $-0,028$ & 0,7830 & 101,4 & 0,021 & 1,180 & 26 \\
\hline & & B & $-0,104$ & 0,3299 & 102,4 & 0,235 & 1,360 & 29 \\
\hline & \multirow{2}{*}{$3 \mathrm{~A}$} & $\mathrm{~A}$ & $-0,069$ & 0,5225 & 100,1 & 0,109 & 1,600 & 34 \\
\hline & & B & $-0,133$ & 0,2951 & 101,2 & 0,266 & 1,430 & 30 \\
\hline
\end{tabular}

Fonte: Os autores

Uma análise mais abrangente foi realizada utilizando os dados dos três lotes armazenados em mesma condição. A primeira hipótese testada foi a de igualdade do coeficiente angular da regressão linear individual dos lotes em mesma condição de embalagem. Caso a hipótese nula $\left(\mathrm{H}_{0 \beta}: \beta_{1}=\beta_{2}=\beta_{3}\right)$ seja rejeitada em um nível de significância de $25 \%$, o agrupamento dos dados em um coeficiente angular estimado comum não é possível (WESSELS et al., 1997), caso isso ocorra, nenhuma informação adicional da ANCOVA deve ser levada em conta e a avaliação deve ficar restrita ao pior lote.
Nas análises de covariância apresentadas nas Tabelas 3 e 4, a interação lote*tempo representa a comparação entre os três lotes do mesmos compostos em mesma condição de embalagem.

Observa-se que o $p$-valor é maior que 0,25 , portanto não existe uma diferença significativa entre os três lotes em mesma condição de embalagem. $\mathrm{O} p$-valor do produto embalado em PVDC indica que existe uma dependência negativa do teor dos dois compostos em relação ao tempo, enquanto o coeficiente angular do produto embalado em PVDC + FP indica uma não dependência do produto em relação ao tempo. 
Tabela 3 - Análise de covariância para o composto A e B do produto embalado em PVDC

\begin{tabular}{|c|c|c|c|c|c|c|}
\hline \multirow{5}{*}{ Composto A } & C.V. & g.l. & SQ & QM & $\mathbf{F}$ & $\operatorname{Pr}(>\mathbf{F})$ \\
\hline & Lote & 2 & 13,5630 & 6,7820 & 3,25 & 0,074 \\
\hline & Tempo & 1 & 41,0420 & 41,0420 & 19,66 & 0,001 \\
\hline & Lote*Tempo & 2 & 0,3040 & 0,1520 & 0,07 & 0,930 \\
\hline & Resíduo & 12 & 25,0550 & 2,0880 & & \\
\hline \multirow{5}{*}{ Composto B } & C.V. & g.l. & SQ & QM & $\mathbf{F}$ & $\operatorname{Pr}(\mathbf{F})$ \\
\hline & Lote & 2 & 13,8478 & 6,9239 & 3,54 & 0,062 \\
\hline & Tempo & 1 & 2,2441 & 2,2441 & 1,15 & 0,305 \\
\hline & Lote*Tempo & 2 & 0,3002 & 0,1501 & 0,08 & 0,927 \\
\hline & Resíduo & 12 & 23,4507 & 1,9542 & & \\
\hline
\end{tabular}

Fonte: Os autores

Tabela 4 - Análise de covariância para o composto A e B do produto embalado em PVDC +FP

\begin{tabular}{|c|c|c|c|c|c|c|}
\hline \multirow{5}{*}{ Composto A } & C.V. & g.l. & SQ & $\mathbf{Q M}$ & $\mathbf{F}$ & $\operatorname{Pr}\left(F_{)}\right.$ \\
\hline & Lote & 2 & 15,2340 & 7,6170 & 12,78 & 0,001 \\
\hline & Tempo & 1 & 36,625 & 36,6250 & 61,41 & $<0,001$ \\
\hline & Lote* Tempo & 2 & 1,3470 & 0,6740 & 1,13 & 0,355 \\
\hline & Resíduo & 12 & 7,1560 & 0,5960 & & \\
\hline \multirow{5}{*}{ Composto B } & C.V. & g.l. & SQ & QM & $\mathbf{F}$ & $\operatorname{Pr}(>F)$ \\
\hline & Lote & 2 & 15,8578 & 7,9289 & 3,71 & 0,056 \\
\hline & Tempo & 1 & 6,1608 & 6,1608 & 2,88 & 0,115 \\
\hline & Lote*Tempo & 2 & 0,5650 & 0,2825 & 0,13 & 0,878 \\
\hline & Resíduo & 12 & 25,6676 & 2,1390 & & \\
\hline
\end{tabular}

Fonte: Os autores

A última propriedade a ser avaliada é a igualdade

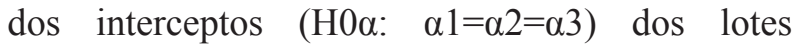
acondicionados em mesma condição. Os dados indicam a rejeição da hipótese

nula (p-valor $<0.25$ ). Portanto, um modelo de coeficiente angular e intercepto comum não pode ser utilizado, ficando restrito a um modelo de coeficiente angular comum e interceptos diferentes, sendo escolhido o pior caso para ambas as embalagens.

As Figuras 3 e 4 correspondem às regressões lineares de coeficiente angular comum e intercepto diferente para os compostos A e B do produto $\mathrm{X}$ embalado em PVDC e as Figuras de 5 e 6 correspondem às regressões lineares de coeficiente angular comum e intercepto diferente para os compostos $\mathrm{A}$ e $\mathrm{B}$ do produto $\mathrm{X}$ embalado em PVDC+FP.
Pode-se perceber que o composto A é o fator determinante estabilidade desse produto $\mathrm{e}$ comparando-se os resultados obtidos para o produto embalado em PVDC o prazo de validade esperado é menor quando comparado ao produto embalado em PVDC+FP, 28 meses para PVDC contra 32 meses para $\mathrm{PVDC}+\mathrm{FP}$, demonstrando novamente a eficiência dessa embalagem.

\section{AVALIAÇÃO DOS PRODUTOS DE DEGRADAÇÃO}

As substâncias relacionadas derivadas da degradação dos componentes principais do comprimido também são consideradas atributos críticos susceptíveis de influência à qualidade do produto. A avaliação anterior indicou que $\mathrm{o}$ composto responsável pela estimação do prazo de validade desse produto é o composto A. Portanto, a avaliação será feita sobre o principal produto de 
degradação desse composto e será denominada aqui como impureza A.

A Tabela 5 apresenta os resultados de teor da Impureza $\mathrm{A}$ obtidos para os lotes do produto $\mathrm{X}$

Figura 3 - Regressão linear do Composto A do produto $\mathrm{X}$ embalado em PVDC 90

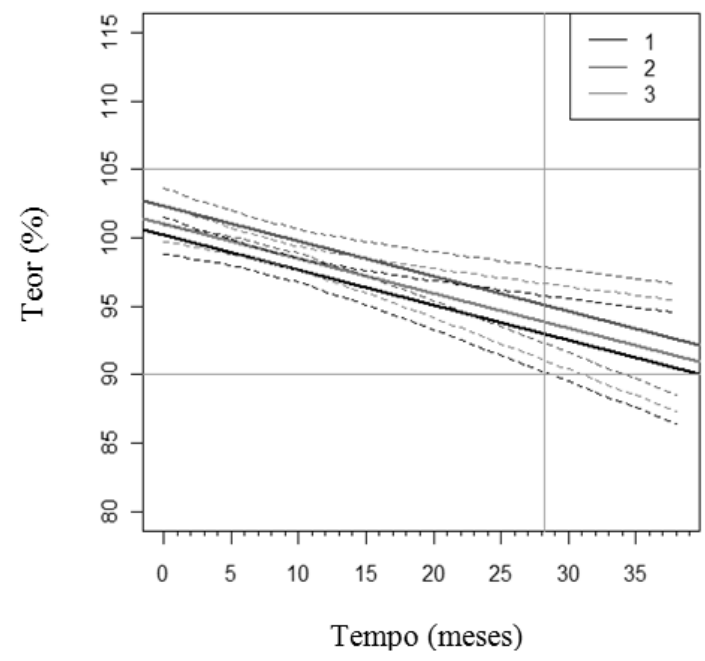

Figura 5 - Regressão linear do Composto A do produto $\mathrm{X}$ embalado em PVDC+FP

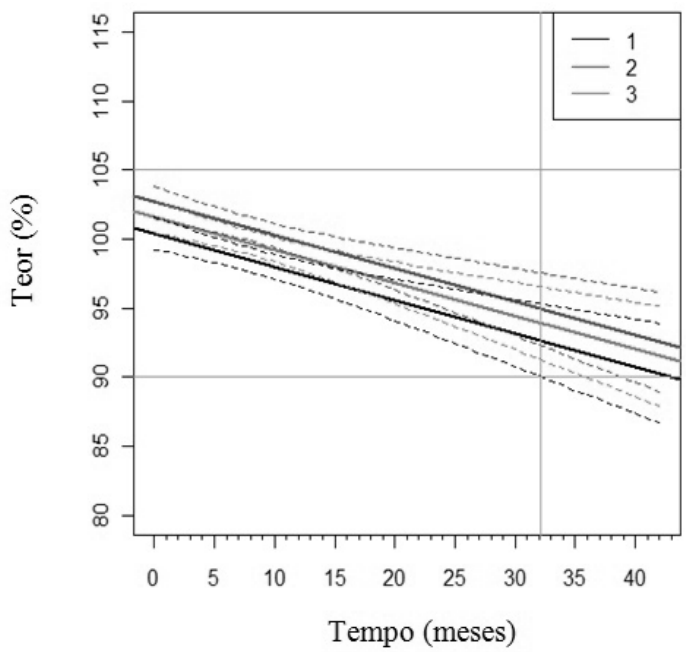

Nesse caso, com um nível de significância de $25 \%$, pode-se afirmar que em ambas as embalagens os coeficientes angulares e interceptos não diferem significativamente entre si, podendo-se utilizar um modelo linear com intercepto e coeficiente angular comum para o produto em mesma condição de durante os 18 meses de estudo de estabilidade e as Tabelas 6 e 7 apresentam a análise de covariância dos dados de substância relacionadas do produto embalado em PVDC e PVDC + FP, respectivamente.

Figura 4 - Regressão linear do Composto B do produto $\mathrm{X}$ embalado em PVDC 90

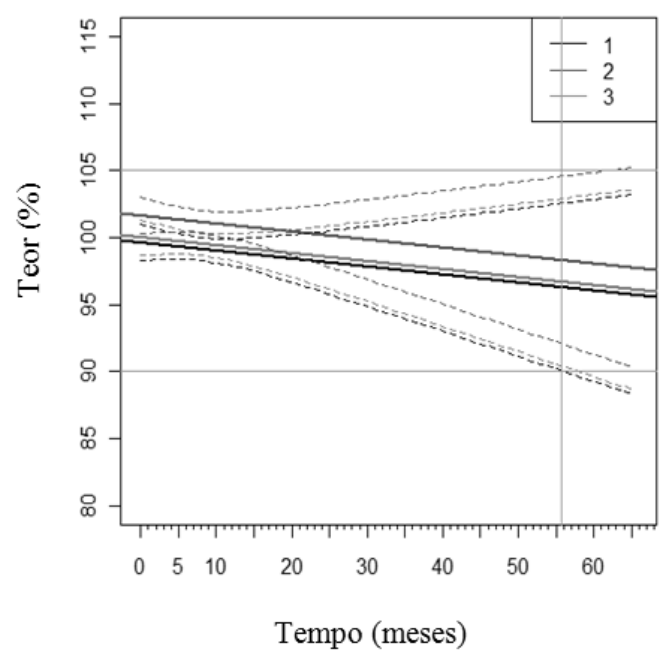

Figura 6 - Regressão linear do Composto B do produto $\mathrm{X}$ embalado em PVDC +FP

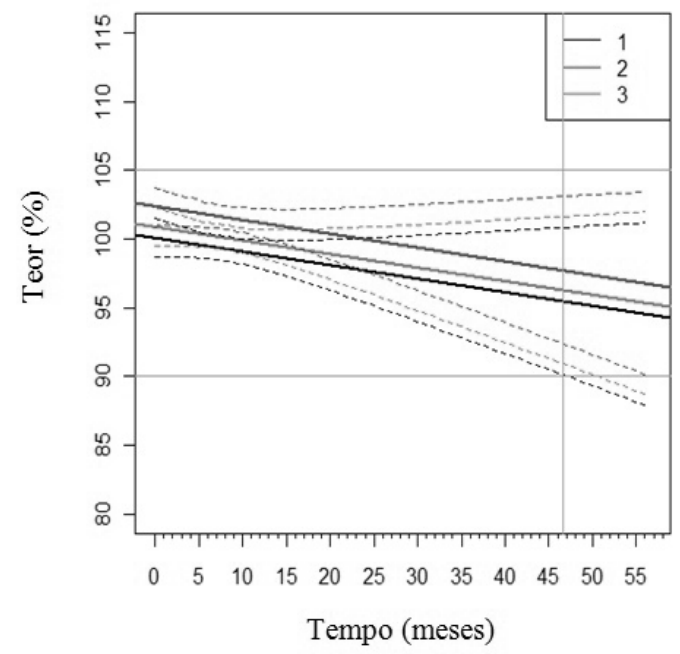

embalagem. Dessa forma, os modelos lineares foram descritos, e os resultados estão na Tabela 8.

A partir da modelagem pode-se perceber que apesar do crescimento de impurezas no produto final ser significativa a uma taxa de $0,37 \%$ ao ano para o produto em PVDC e $0,15 \%$ ao ano 
para o produto em PVDC $+\mathrm{FP}$, essa impureza não é o fator que determina o prazo de validade do produto, isso porque a avaliação está sendo feita em apenas um derivado de degradação do composto determinante do prazo de validade e durante o estudo de estabilidade outros produtos de degradação são gerados tanto do composto A como do composto B.

Tabela 5- Resultado do teor da impureza A do produto X embalado em PVDC 90 e PVDC 90+FP em estudo de estabilidade de longa duração $\left(30^{\circ} \mathrm{C} / 75 \%\right.$ U.R.)

\begin{tabular}{cc|cccccc}
\hline \multicolumn{7}{c}{} & \multicolumn{7}{c}{ Tempo (meses) } \\
\hline Embalagem & Lote & 0 & 3 & 6 & 9 & 12 & 18 \\
\hline \multirow{3}{*}{ PVDC 90 } & 1 & $<0,10$ & $<0,10$ & 0,20 & 0,23 & 0,34 & 0,55 \\
& 2 & $<0,10$ & $<0,10$ & $<0,10$ & 0,21 & 0,32 & 0,51 \\
& 3 & $<0,10$ & $<0,10$ & 0,10 & 0,24 & 0,32 & 0,50 \\
& 1A & $<0,10$ & $<0,10$ & $<0,10$ & 0,16 & 0,12 & 0,18 \\
PVDC 90+FP & 2A & $<0,10$ & 0,10 & 0,10 & $<0,10$ & 0,12 & 0,26 \\
& 3A & $<0,10$ & $<0,10$ & $<0,10$ & 0,18 & 0,14 & 0,24 \\
\hline
\end{tabular}

Fonte: Os autores

Tabela 6- Análise de covariância para a Impureza A do produto embalado em PVDC 90

\begin{tabular}{lccccr}
\hline \multicolumn{1}{c}{ C.V } & g.l & SQ & QM & F & Pr( $(\mathbf{F})$ \\
\hline Intercepto & 2 & 0,0066 & 0,0033 & 1,21 & 0,331 \\
Tempo & 1 & 0,6098 & 0,6098 & 224,8 & $<0,001$ \\
Lote* Tempo & 2 & 0,0004 & 0,0002 & 0,064 & 0,938 \\
Resíduo & 12 & 0,0325 & 0,0027 & & \\
\hline
\end{tabular}

Fonte: Os autores

Tabela 7- Análise de covariância para a Impureza A do produto embalado PVDC 90 +FP

\begin{tabular}{|c|c|c|c|c|c|}
\hline C.V. & g.l. & SQ & QM & $\mathbf{F}$ & $\operatorname{Pr}\left({ }_{F}\right)$ \\
\hline Intercepto & 2 & 0,0014 & 0,001 & 0,22 & 0,808 \\
\hline Tempo & 1 & 0,1006 & 0,101 & 31,66 & $<0,001$ \\
\hline Lote*Tempo & 2 & 0,0017 & 0,002 & 0,27 & 0,767 \\
\hline Resíduo & 12 & 0,0381 & 0,003 & & \\
\hline
\end{tabular}

Fonte: Os autores

Tabela 8 - Parâmetros do modelo linear para as substâncias relacionadas ao composto A do Produto X.

\begin{tabular}{cccc}
\hline & $\alpha$ & $\beta$ & $\begin{array}{c}\text { Prazo estimado } \\
\text { (meses) }\end{array}$ \\
\hline PVDC 90 & $-0,053$ & 0,03111 & 120 \\
PVDC +FP & $-0,012$ & 0,01263 & 59 \\
\hline
\end{tabular}

Fonte: Os autores 
Uma avaliação da taxa de formação dessa impureza permite concluir que como já identificado no item 3.1, a embalagem Flow Pack fornece uma proteção maior a esse composto, diminuindo significativamente a taxa de formação desse produto de degradação.

\section{Considerações finais}

$\mathrm{O}$ uso de modelos lineares para a avaliação de dados de estabilidade do produto $\mathrm{X}$ permitiu verificar que a embalagem PVDC + FP é significativamente mais eficiente que a embalagem de PVDC. Entretanto, outros fatores devem ser levados em consideração na escolha da embalagem do produto final, pois apesar da eficiência aumentar quando a embalagem Flow Pack é utilizada, o custo desse tipo de embalagem é mais alto quando comparado ao PVDC, o que poderia acarretar um aumento significativo no custo do produto final.

Normalmente, o modelo linear é aplicado ao estudo de estabilidade para estimar a taxa de degradação e/ou formação de impurezas do produto. Porém, nesse trabalho foi possível verificar que a análise de regressão linear pode ser uma ferramenta muito útil no estudo de dados de estabilidade, permitindo avaliar a diferença de eficiência de duas embalagens na proteção do produto e estabelecer uma série de correlações entre os dados obtidos durante o estudo.

\section{Referências}

BAR, R. Statistical evaluation of stability data: Criteria for change-over-time and data variability. PDA Journal of Pharmaceutical Science and Technology, Bethesda, v. 57, n. 5, p. 369-377, Sep. 2003.

BRASIL. Ministério da Saúde. Agência Nacional de Vigilância Sanitária. Resolução - RE no 398, de 12 de novembro de 2004. Determina a publicação do Guia para Realização de Estudo de Estabilidade. Diário Oficial da União, Brasília, 2004.
CHOW, S. C. Statistical Design and Analysis of stability Study. Duhan: Chapman and Hall, 2007.

CHOW, S. C.; SHAO, J. Test for batch-to-batch variations in stability analysis. Statistics in Medicine, Chichester, v. 8, n. 7, p. 883-890, jul. 1989.

International Conference on Harmonisation (ICH). Guidance for industry Q1A (R2) stability testing of new drug substances and products. 2003. Disponível em: <http://www.ich.org/LOB/media/MEDIA415.pdf $>$. Acesso em: 17 nov. 2010.

LEE, H.Y.; WU, P.C.; LEE, Y.J. Stab: an R package for drug stability data analysis. Computer methods and Programs in Biomedicine, Kaohsiung City, v. 100, n. 2, p. 140-148, abr. 2010.

NORWOOD, T. E. Statistical analysis of pharmaceutical stability data. Drug Development and Industrial Pharmacy, New Jersey, v. 12, n. 4, p. 553-560, jan. 1986.

TEAM RDC. R: A language and environment for statistical computing. R Foundation for Statistical Computing. Vienna, 2011. Disponivel em: <http:// www.R-project.org>. Acesso em: 17 nov. 2010.

TSONG, Y.; CHEN, W.-J.; CHEN, C. W. ANCOVA Approach for shelf life analysis of stability study of multiple factor designs. Journal of Biopharmaceutical Statistics, New York, v. 13, n. 3, p. 375-393, jun. 2003.

WESSELS, P.; HOLZ, M.; ERNI, F.; KRUMMEN, K.; OGORKA, J. Statistical evaluation of stability data of pharmaceutical products for specification setting. Drug Development and Industrial Pharmacy, London, v. 23, n. 5, p. 427-439, jan. 1997.

Recebido em 4 Outubro 2012 - Received on October 4, 2012. Aceito em 4 Março, 2013 - Accepted on March 4, 2013. 
